\title{
Nanotechnology-Based Histone Deacetylase Inhibitors for Cancer Therapy
}

\author{
Bin Tu ${ }^{1,2}$, Meng Zhang ${ }^{1 *}$, Tuanbing Liu ${ }^{1}$ and Yongzhuo Huang ${ }^{1,2,3 *}$ \\ 1 State Key Laboratory of Drug Research, Shanghai Institute of Materia Medica, Chinese Academy of Sciences, Shanghai, \\ China, ${ }^{2}$ University of Chinese Academy of Sciences, Beijing, China, ${ }^{3}$ NMPA Key Laboratory for Quality Research \\ and Evaluation of Pharmaceutical Excipients, Beijing, China
}

\section{OPEN ACCESS}

Edited by:

Christiane Pienna Soares,

São Paulo State University, Brazil

Reviewed by:

Na Zhang,

Shandong University, China Salman Akhtar,

Integral University, India

Flavio Rizzolio,

Ca' Foscari University of Venice, Italy

Christophe Blanquart,

Centre National de la Recherche

Scientifique, France

${ }^{*}$ Correspondence:

Meng Zhang

Zhangmeng3@simm.ac.cn

Yongzhuo Huang

yzhuang@simm.ac.cn

Specialty section:

This article was submitted to Epigenomics and Epigenetics, a section of the journal Frontiers in Cell and Developmental Biology

Received: 07 March 2020 Accepted: 01 May 2020

Published: 03 June 2020

Citation:

Tu B, Zhang M, Liu T and Huang Y (2020)

Nanotechnology-Based Histone Deacetylase Inhibitors for Cancer Therapy. Front. Cell Dev. Biol. 8:400.

doi: 10.3389/fcell.2020.00400
Histone deacetylase inhibitors (HDACi) have been approved and achieved success in hematologic malignancies. But its application in solid tumors still confronts big challenges and is hampered by low treatment efficacy. Nanotechnology has been widely applied in cancer therapy, and nanomedicine could improve drug stability, prolong the circulation half-life, and increase intratumoral drug accumulation. Therefore, nanomedicine is a promising strategy to enhance HDACi therapy efficacy. The review provides a summary of the advances of HDACi nanomedicines with a focus on the design principles of the targeting delivery systems for HDACi.

Keywords: Histone deacetylase inhibitors (HDACi), nanotechnology, nanomedicine, cancer therapy, solid tumor, targeting drug delivery, combination therapy

\section{INTRODUCTION}

Histone deacetylase inhibitors (HDACi) prevent histone deacetylases from removing acetyl group from the lysine amino acid of histone and thus open the chromatin structure to promote the access to transcription factors and facilitate gene transcription, consequently regulating cell proliferation (Figure 1A). HDACi exert anticancer mechanisms of HDACi include cell cycle arrest, apoptosis, autophagy, anti-angiogenesis, and regulation of epithelial-mesenchymal transition and immune responses (Eckschlager et al., 2017; Wawruszak et al., 2019). United States FDA has approved five HDACi for hematologic malignancies, including vorinostat (2006), romidepsin (2009), belinostat (2014), chidamide (2015), and panobinostat (2015).

However, there is limited success in solid tumor treatment, which could account for various reasons. First, treatment efficacy is affected by the poor pharmacokinetics such as short half-life, and fast metabolism and clearance; second, low specificity often causes off-target and side effects; third, low solubility and tissue/cell permeability of HDACi limit intratumor delivery; and fouth, drug resistance is readily developed (Li and Seto, 2016; Suraweera et al., 2018). Targeted delivery and controlled drug release could be a potential solution to these issues in HDACi-based therapy.

Nanomedicine is referred to as a specific size - "about 100 nanometers or less - that biological molecules and structures operate in living cells" $(\mathrm{NIH})$, but often referred to a wider scale - less than $1000 \mathrm{~nm}$. Cancer nanomedicines can improve efficacy and reduce side-toxicity by increasing drug solubility, improving the pharmacokinetic profiles, and enhancing intratumoral drug delivery (Bayda et al., 2018; Palazzolo et al., 2018).

For example, rational design of nanoparticles can enhance drug accumulation and cellular uptake in the tumor by fine-tuning size and surface property. Small size can increase intratumoral infiltration because nanoparticles with size between 20 and $60 \mathrm{~nm}$ are more likely to penetrate deep 
tumor tissues via the enhanced permeability and retention (EPR) effect and uptaken by tumor cells, while the larger is easily captured by the reticuloendothelial system (RES) and the smaller less than $5 \mathrm{~nm}$ cleared by renal (Bayda et al., 2018).

\section{NANOTECHNOLOGY-BASED PLATFORMS OF HDACI FOR CANCER THERAPY}

There are two strategies for tumor-targeting delivery - passive and active targeting. Nanomedicine could preferentially accumulate into the tumors to achieve passive targeting through EPR effect, by which the macromolecules or nanomaterials tend to distribute in the tumor due to the leaky neovasculature and poor lymphatic drainage (Maeda, 2012; Ahmad et al., 2019). EPR effect is typically observed in rodent tumor models, but with limited evidence in human patients. It is believed that the EPR effect is highly variable due to the heterogeneity of the tumors (Danhier, 2016). Interestingly, a recent study found that $97 \%$ of PEGylated AuNPs entered tumors via active trans-endothelial pathways, while inter-endothelial gaps accounted for a very small proportion (Sindhwani et al., 2020). However, the conclusion was drawn based on the non-deformable AuNPs and needs further support by using various nanoparticles including the deformable vesicles.

Active-targeting delivery represents another common strategy. The general design is based on ligand/receptor-specific interaction (e.g., trastuzumab and HER-2, folate and folate receptors): the modified ligands on nanomedicine and the receptors expressed on cancer cells or other cells in tumor microenvironment (TME) (Figure 1B). Certain nanomaterials can selectively bind with the receptors on cancer cells, e.g., the interaction of albumin nanoparticles and albumin-binding proteins (Lin et al., 2016; Zhao et al., 2018a).

\section{Nanotechnology-Based Passive Targeting}

Tumor vessels are characterized by the abnormal vasculatures with the leaky vessel walls that lack tight junction between endothelial cells and pericytes. The leakage facilitates nanomedicine to permeate and accumulate in the tumor tissues, compared to the normal tissues with 5-10 $\mathrm{nm}$ endothelial junctions (Hobbs et al., 1998). The preferential accumulation of drugs in the tumor means the lower drug dose and fewer side effects. Moreover, nanomedicines can increase the intracellular drug retention based on the "size-exclusion effect" that reduces the transporters-mediated drug efflux and reverse drug resistance (Liu et al., 2012), because P-glycoproteins can only recognize the substrate molecules less than $2000 \mathrm{Da}$ (Seelig, 1998).

Nanotechnology-based delivery can improve anti-tumor efficacy of HDACi by overcoming their disadvantages. For example, poor solubility is a problem for many HDACi. Starch is a polysaccharide widely used in pharmaceutics due to its biocompatibility and biodegradability. Encapsulating CG-1521 (an HDACi) into the starch nanoparticles via the emulsion-solvent diffusion method could increase its water solubility and cellular uptake, leading to enhanced anti-tumor activity (Alp et al., 2019). Panobinostat has been explored for glioma treatment. However, the poor solubility prevents its administration by convection-enhanced delivery (CED) in brain cancer. To solve this problem, an HDACi nano-system composed of poloxamer 407 (P407) was designed (Singleton et al., 2017). P407 can self-assemble into nano-micelles based on its hydrophobic polypropylene glycol chain in the middle and two hydrophilic PEG chains at both ends. The panobinostatloaded P407 micelles increased the intracranial panobinostat concentration by skirting the blood-brain barrier (BBB), resulting in glioma repression and prolonged survival of the high-grade glioma-bearing rats by one CED dose.

Poly(D, L-lactide-co-glycolide) (PLGA) is an injectable polymer approved by FDA. The PEG-lecithin coated PLGA nanoparticles encapsulating vorinostat or quisinostat were prepared via the single-step nanoprecipitation method; the delivery systems achieved the controlled release and suppressed the DNA double-strand break (DSB) repair in tumor cells, serving as the potential radiosensitizers and exhibiting synergistic effect in the mouse xenograft models of colorectal and prostate carcinomas via a mechanism of the prolongation of $\gamma-\mathrm{H} 2 \mathrm{AX}$ foci (Wang et al., 2015).

Owing to the existence of the bladder permeability barrier $(\mathrm{BPB})$, drugs are difficult to distribute in the bladder tumor. The nano delivery strategy through the EPR effect could not help much. To address this problem, poly(guanidinium oxanorbornene) (PGON), a novel cell-penetrating polymer, was used to modify the belinostat-loaded PLGA nanoparticles to improve the BPB-permeability (Martin et al., 2013). The PGONmodified nanoparticles enhanced the penetration in the mouse bladder more than 10 folds compared to the nanoparticles without PGON, and significantly enhanced intracellular uptake and anti-tumor activity.

Furthermore, it has been reported that the biodegradable poly(DL-lactide-co-glycolide)/poly(ethylene glycol) (LGE) block copolymer could encapsulate vorinostat by using the nanoprecipitation method (Kwak et al., 2015). The vorinostatloaded nanoparticles promoted the intensive accumulation in the tumors and exhibited the enhanced antitumor activity in the subcutaneous cholangiocarcinoma-bearing mice compared to free vorinostat.

\section{Nanotechnology-Based Active Targeting}

There have been various active-targeting nanoparticles developed for HDACi therapy. Ligand-modified nanoparticles facilitate the tumor-targeting and cell internalization via specific interaction between the ligand decorated on nanoparticles and the corresponding receptors overexpressed on the tumor cells. For example, the nutrient transporters (e.g., albumin-binding protein) are often overexpressed in tumor cells due to their hunger for energy and thus serve as the targeting delivery receptors (Zhao et al., 2018b). The active targeting ligand molecules mainly include small molecules, peptides, antibodies, and aptamers (Bazak et al., 2015). 

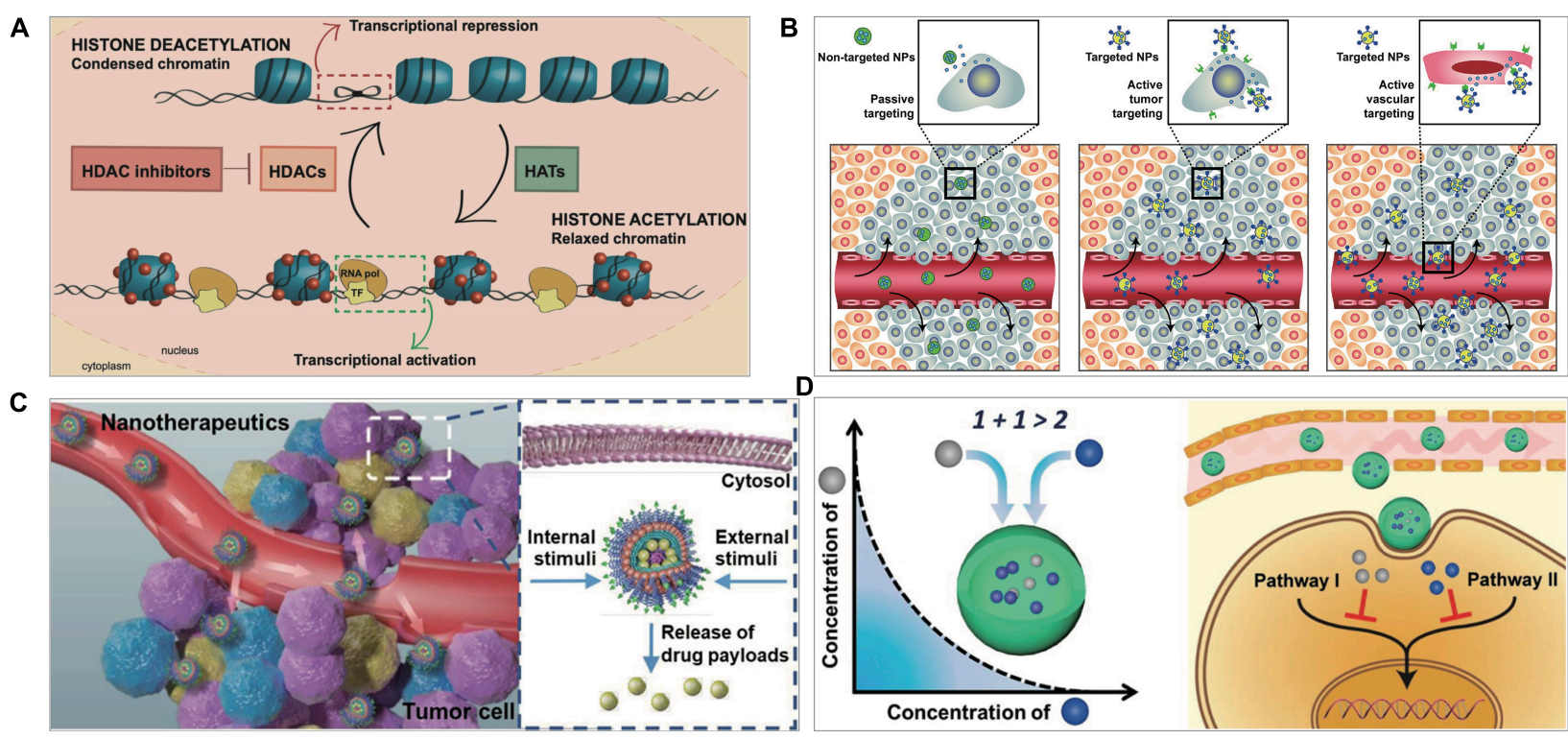

FIGURE 1 | (A) HDACi effect on chromatin remodeling. HDACs deacetylate histones and inhibit gene transcription and histone acetyltransferases (HATs) acetylate histones and activate transcription. HDACi inhibit HDACs, and thus maintain an open chromatin conformation (San Jose-Eneriz et al., 2019).

(B) Nanotechnology-based targeting delivery (Farokhzad and Langer, 2009). (C) Stimuli-responsive controlled release (Qiao et al., 2019). (D) Nanotechnology-based combination (Ma et al., 2013). Figures are reproduced with permission from the publishers of the cited references.

\section{Small Molecular Ligand-Mediated Active Targeting}

Small molecules are advantageous for their low price, small size, and chemical stability. For example, tumor-associated macrophages (TAM) overexpress mannose receptors and are ideal targets for cancer treatment. Mannose is a specific ligand of the mannose receptor and can be chemically modified to the surface of liposomes to deliver vorinostat for targeting TAM (Peng et al., 2017). Triple-negative breast cancer (TNBC) cells often overexpress lysophosphatidic acid receptor 1 (LPAR1) and G2A receptors. By modification with LPA (a ligand of LPAR1) and LPC (a ligand of G2A), the PEGylated nanoemulsions of panobinostat and decitabine were prepared via the solvent injection method (Kim et al., 2019). The cellular uptake and in vivo biodistribution of the nanoemulsions was associated with the LPAR1 expression and the optimal modification ratio of LPA to LPC was 1:1. Furthermore, the folic acid (FA)-modified dendrimer-HDACi conjugates were prepared by click reaction between the azido-modified suberoylanilide hydroxamic acid (SAHA) and polyamidoamine (PAMAM) (Zong et al., 2015). FA mediated tumor-targeting delivery of the conjugates and SAHA remained inactivity until released from the conjugates in the cancer cells.

\section{Peptide/Protein Ligand-Mediated Active Targeting}

Peptides and proteins are the commonly used ligands. For example, transferrin receptors (TfR) are often overexpressed in many malignant cells for iron uptake (Lai et al., 2009; Amreddy et al., 2018), which is a useful target for cancer drug delivery. A TfR-targeted lipid-protein hybrid nano-platform was designed by encapsulating the vorinostat/paclitaxel co-loading albumin nanoparticles into the transferrin-modified liposomes (Ruttala et al., 2017). The liposomes effectively protected the drugs from rapid elimination during the circulation and the surfaceanchored transferrin could bind with TfR on the cancer cells with 8-10 folds of higher efficiency compared with non-targeted nanoparticles. The nano-platform yielded enhanced efficacy in a HepG-2 xenografted mouse model via vorinostat-sensitized paclitaxel chemotherapy.

\section{Antibody Ligand-Mediated Active Targeting}

$\mathrm{EGFR}^{\mathrm{T} 790 \mathrm{M}}$ secondary mutation is readily developed in those receiving gefitinib therapy in non-small cell lung cancer (NSCLC), thus resulting in gefitinib resistance. A dualtargeted liposome system for codelivery of gefitinib and vorinostat has been prepared by chemical modification with anti-HER-2 antibody and mannose (Peng et al., 2017). The liposomes can target the HER-2-overexpressing tumor cells and mannose receptor-expressed TAM, respectively. The dualtargeted liposomes reversed the resistance of $\mathrm{EGFR}^{\mathrm{T} 790 \mathrm{M}_{-}}$ positive NSCLC to gefitinib via reprogramming the TAMs and regulating the ROS/NOX3/MsrA axis of cancer cells (Figure 2B) (Peng et al., 2017).

\section{Stimuli-Responsive Controlled Drug Release}

After nanomedicine reaches the tumor site, the effectively controlled release of drugs becomes a key issue. The drug release rate can significantly affect therapeutic efficacy. For example, fast intracellular drug release was beneficial to overcome chemoresistance and kill the tumor cells (Gao et al., 2011). The stimuli-responsive strategy provides a useful method for achieving site-specific controlled drug release. 


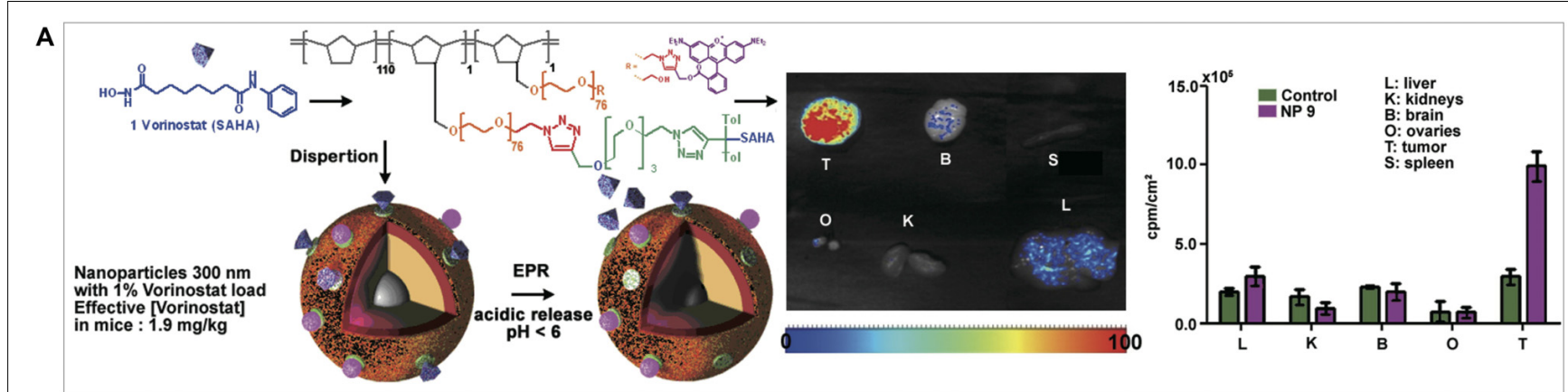

B

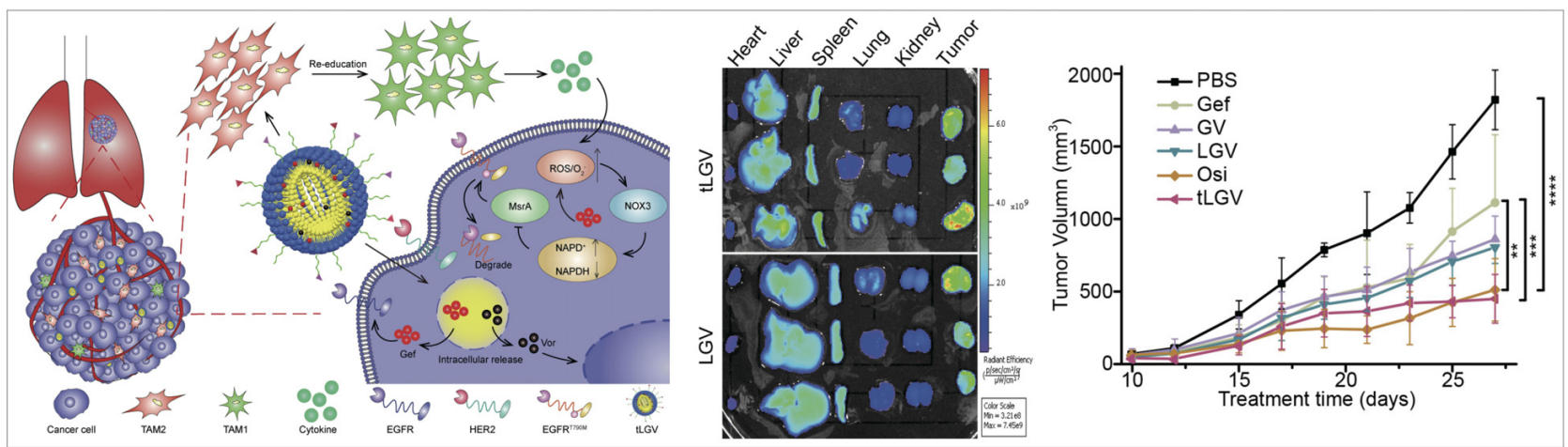

C

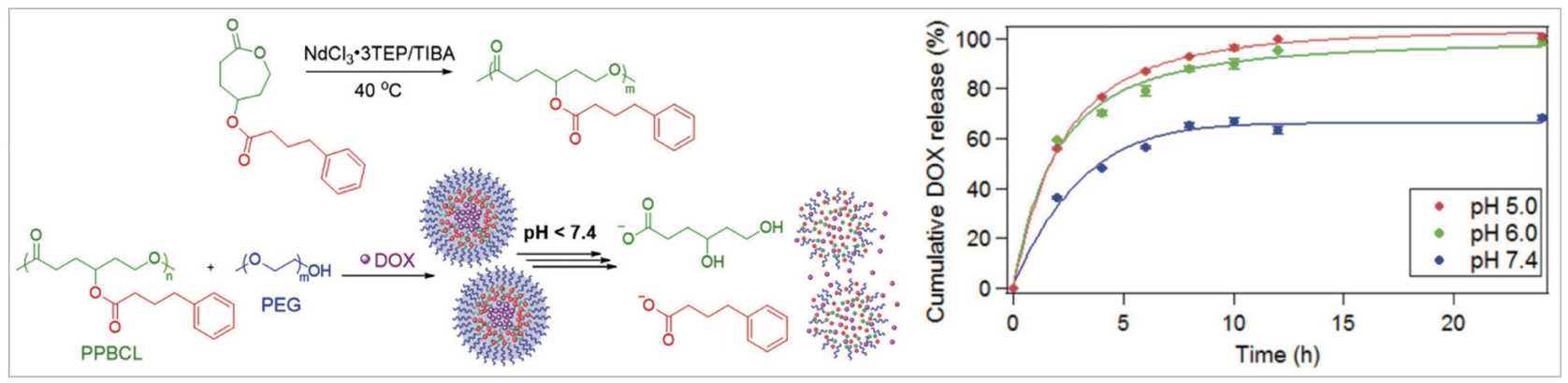

D
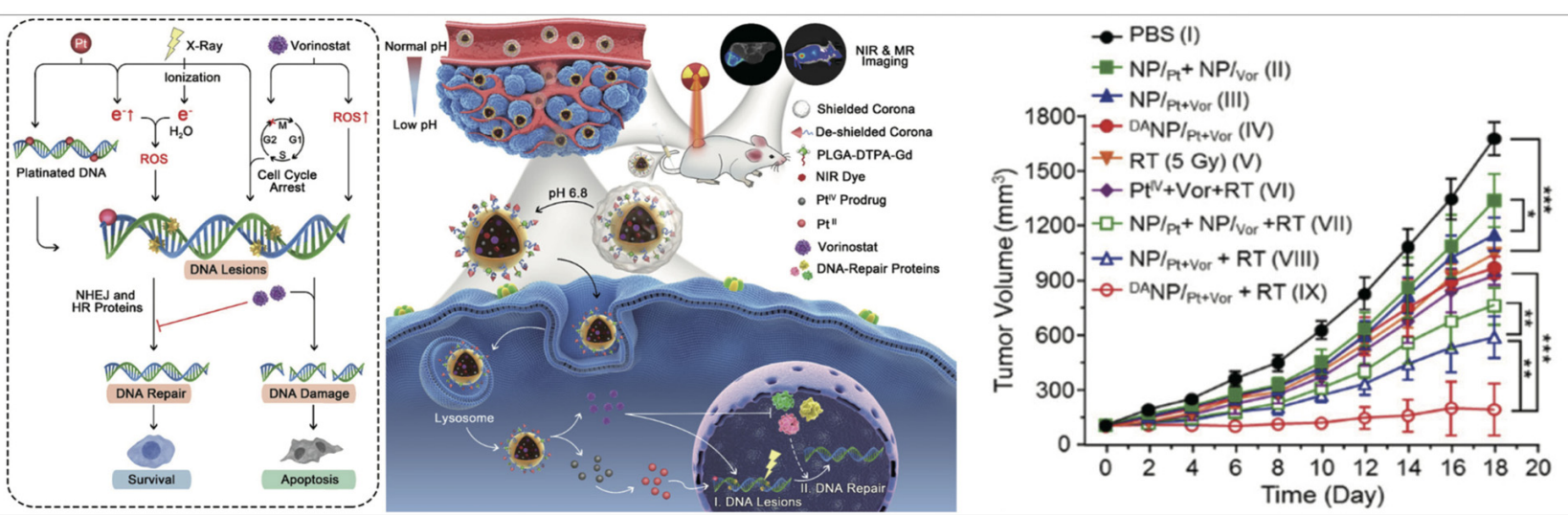

FIGURE 2 | Application examples of nanotechnology-based HDACi therapy. (A) Passive targeting: the scheme of vorinostat-polymer conjugate nanoparticles and the enhanced tumor accumulation via the EPR effect (Denis et al., 2014). (B) Active targeting: the scheme of dual-targeted liposome system co-loading gefitinib and vorinostat and targeting HER-2 expressed tumor cells and mannose receptor expressed TAMs, its anti-tumor effect on H1975 tumor mouse model, and the accumulation by in vivo imaging study (Peng et al., 2017). (C) Stimuli-responsive controlled drug release: the illustration of pH-responsive DOX loading nanoparticles by the self-assembling of PBA conjugated polycaprolactone, and the drug release in response to different pH (Kularatne et al., 2018). (D) Combination therapy: the scheme of vorinostat-containing nanoparticles for sensitizing radiotherapy (Jiang et al., 2018). ${ }^{*} p<0.05$ was considered statistically significant in all analysis (95\% confidence level). ${ }^{\star} p<0.05,{ }^{\star \star} p<0.01,{ }^{* \star} p<0.001$, and ${ }^{* \star \star \star} p<0.0001$. Figures are reproduced with permission from the publishers of the cited references. 
Tumor microenvironment is characterized by its abnormal conditions, like acidic $\mathrm{pH}$, enhanced ROS or GSH, and overexpressed proteases, which serve as endogenous triggers to stimulate drug release from nanomedicines (Chen et al., 2016). Besides, exogenous stimuli can also be used for controlled drug release, such as light, ultrasound, and external alternating magnetic field (Patra et al., 2018). Various stimuli-responsive nanomedicines have been developed for triggering drug release at the tumor (Figure 1C).

\section{Redox-Responsive Nanoparticles}

There are excessive GSH and/or ROS in cancer cells (Weinberg et al., 2019). Disulfide linkage that can be cleaved by GSH via reduction reaction has been widely used in stimuli-responsive delivery. An amphiphilic prodrug SAHA-S-S-VE was prepared via conjugation of vorinostat (SAHA) with vitamin $\mathrm{E}$ (VE) through a disulfide bond, which could self-assemble into the nanoparticles with addition of $\mathrm{D}$-a-tocopheryl polyethylene glycol succinate (TPGS). The prodrug nanoparticles could release vorinostat in response to GSH stimulation in a concentrationdependent manner and showed a significant anti-tumor effect in the mice bearing $\mathrm{H} 22$ tumors (Han et al., 2016).

\section{pH-Responsive Nanoparticles}

The acidic $\mathrm{pH}$ ranges from 5-7 in TME caused by the Warburg effect (Dai et al., 2017). Bertrand et al. proposed an interesting ROMP (Ring-Opening Metathesis Polymerization) method for preparing a stealth polymeric nanoparticle platform for delivering HDACi (Bertrand et al., 2019). The alkynemodified cancer drugs can be grafted to the nanoparticles by click chemistry and the chemical bond can be cleaved by acidic $\mathrm{pH}$, thus achieving enhanced intratumor accumulation and controlled release (Figure 2A) (Denis et al., 2014). The $\gamma$ 4-phenylbutyrate- $\varepsilon$-caprolactone monomer was synthesized via modifying the 4-phenyl butyric acid (PBA, an HDACi) with a $\mathrm{pH}$ sensitive ester bond and then polymerized to the homopolymer of poly $(\gamma$-4-phenylbutyrate- $\varepsilon$-caprolactone) (PPBCL) which could self-assemble into nanoparticles with PEG as a surfactant to encapsulate doxorubicin (DOX) (Kularatne et al., 2018). In the acidic environment, the ester bonds were hydrolyzed and the nanoparticles disintegrated and PBA and DOX released (Figure 2C) (Kularatne et al., 2018). Another study showed that various HDACi prodrugs were prepared via linking $n$-dodecanoic acid or cholesterol to K-182, a potent HDACi, with ester bond or disulfide bond; the HDACi prodrugs and a DNA drug were incorporated into the cation nanoparticles, and the hyperacetylation of core histones facilitated the enhanced gene expression (Ishii et al., 2009).

\section{Enzyme-Responsive Nanoparticles}

The overexpressed enzymes in TME are usually associated with cancer progressions, such as matrix metalloproteinases (MMPs), phospholipase A2, and esterase (Fouladi et al., 2017). A typical design is to use an enzyme-substrate sequence or material to conjugate or encapsulate drugs; once cleavage by the tumorassociated enzymes, the drugs would be released. For instance, a hyaluronic acid (HA) derivative (HAPBA) was synthesized via conjugating $\mathrm{PBA}$ to $\mathrm{HA}$ via ester bonds that are susceptible to esterase (Lee et al., 2019). The HAPBA-based nanoparticles increased intratumoral drug accumulation and capture by tumor cells via endocytosis mediated by CD44, the receptor of HA. The intracellular PBA release was triggered by esterase.

\section{Temperature-Responsive Nanogel}

A thermosensitive nanogel system composed of poly(ethylene glycol)-poly(e-caprolactone)-poly(ethylene glycol) (PEG-PCLPEG, PECE) was designed to treat oral squamous cell carcinoma (OSCC) via co-delivering vorinostat and cisplatin (Li et al., 2012). The PECE solution turned into a gel state at body temperature after intratumoral injection. With PECE biodegradation via hydrolysis, drugs were continuously released from the nanogel, yielding enhanced therapeutic effects and reduced side effects.

\section{Nanotechnology-Based Combination Therapy}

Drug combinations can achieve improved therapeutic efficacy with the benefits of reducing side toxicity and drug resistance. HDACi in combination with other anticancer agents have been applied and shown great potential for cancer therapy as a promising solution to the insufficient efficacy of HDACi in solid tumors (Yuan et al., 2017; Suraweera et al., 2018). Yet, a big challenge is the different in vivo fate of the combined drugs; for example, the asynchronous distribution in the tumor may not cause synergistic pharmacological actions in the cancer cells (Zhang et al., 2017). It may be responsible for the inconsistency between the in vitro and in vivo results; many combinations work well in cell tests, but not in vivo. Nanotechnology-based combination therapy can achieve the synchronized pharmacokinetic profile of the combined drugs and facilitate the synchronized delivery (Figure 1D), and has been explored in HDACi therapy.

A prodrug conjugate (VAAP) composed of platinum (IV) prodrug (diaminedichlorodihydroxyplatinum, ACHP) and valproic acid (VA, an HDACi) was synthesized for cancer combination therapy (Yang et al., 2012). VAAP was then loaded into the polyethylene glycol-polycaprolactone (PEG-PCL) micelles. Free Pt (II) and VA were released from the VAAP in cancer cells, with 50-100 times higher cytotoxicity than the simple mixture of ACHP and VA. The PEGPCL/VAAP micelles prolonged drug circulation, and increased intratumoral drug concentration and treatment efficacy in the mice bearing A549 tumors.

A nanomedicine for codelivery of vorinostat and cis, cis, trans- $\left[\mathrm{Pt}(\mathrm{NH} 3)_{2} \mathrm{Cl}_{2}\left(\mathrm{OOC}(\mathrm{CH} 2)_{8} \mathrm{CH} 3\right)_{2}\right]$ was developed, which was characterized by the de-shieldable corona that reduced the capture by the RES but was removed in the acidic TME to expose the cell-penetrating peptides (Jiang et al., 2018). The cell-penetrating peptide facilitated the nanomedicine into the tumor cells and the released $\mathrm{Pt}$ chelated into DNA and the released vorinostat sensitized the cancer cells by promoting ROS production and inhibiting DAN repair proteins (Figure 2D). Therefore, the nanotechnology-based codelivery yielded a synergistic effect on radiotherapy sensitization. 
Another combination therapy strategy is to co-administrate the single drug-loaded nanoparticles with a benefit of fine-tuned proportion. The nanogels were prepared from the polymerization of poly(ethylene glycol) monomethacrylate (POEOMA) to encapsulate vorinostat and etoposide, respectively (Kumar et al., 2018). Both nanogels could release their drugs in response to GSH due to disulfide bond cleavage. The co-administration of vorinostat-loaded nanogel and etoposide-loaded nanogel showed enhanced cytotoxicity in cancer cells.

\section{PERSPECTIVES}

Histone deacetylase inhibitors therapy has achieved great success in hematologic malignancies, but with limited progress in solid tumors. Nanotechnology can improve HDACi efficacy by optimizing drug delivery and controlling drug release. For example, tumor-targeting and stimuli-responsive nanomedicines can be designed to increase the intratumoral accumulation and control drug release in the tumors. HDACi nanomedicines showed an improved anti-tumor efficacy with reduced systemic toxicity. Besides, nanotechnology has also applied to predict the therapeutic efficacy of HDACi and a gold nanoparticle nanosensor was developed for the detection of histone deacetylase (Zhang et al., 2019).

However, nanotechnology-based HDACi still confront several challenges. First, nano delivery systems are inclined to be cleared away by RES, often leading to less than $1 \%$ of the injected nanoparticles reaching to the solid tumors (Wilhelm et al., 2016). The multi-functional targeting strategies will be helpful to overcome the various biological barriers and further increase intratumoral drug accumulation. Because HDACi act in the nucleus, nanotechnology-based nuclear-targeted delivery strategies would enhance treatment efficacy. Second, stimuliresponsive strategies rely on abnormal elements (e.g., acidic $\mathrm{pH})$ in TME. However, in the highly heterogenetic tumors in the human body, the clinical applicability of such endogenous triggers is still unknown and has not been well investigated. Exogenous stimuli (e.g., light, radiofrequency, and ultrasound) could be more controllable than endogenous triggers. The lighttriggered release has lots of reports, but its major problem is poor penetration of light in the human body, rendering limited use in deep tumors such as brain and lung cancers. Radiofrequency and ultrasound are more clinically translational due to the advantages of excellent penetration and energy focus. Yet, Phase III HEAT study using radiofrequency ablation with Lyso-Thermosensitive Liposomal Doxorubicin failed to reach the expected endpoint (Tak et al., 2018). Later, the research team substituted the radiofrequency with an ultrasound trigger for the thermosensitive liposomal doxorubicin and conducted a phase

\section{REFERENCES}

Ahmad, A., Khan, F., Mishra, R. K., and Khan, R. (2019). Precision cancer nanotherapy: evolving role of multifunctional nanoparticles for cancer active targeting. J. Med. Chem. 62, 10475-10496. doi: 10.1021/acs.jmedchem.9b0 0511
I clinical trial (Lyon et al., 2018; Gray et al., 2019). Therefore, the clinical translation for stimuli-responsive methods still needs a lot of further investigations. Third, large-scale production of nanoparticles with consistent quality control is an underexplored issue; currently, most publications focus on the laboratory scale.

Moreover, nanotoxicity is a major issue in developing drug delivery systems and is highly related to nanomaterials. The success of many marked nanomedicines has eased the concerns. Liposomes and polymeric micelles have been demonstrated to be highly druggable and biocompatible. However, the nonbiodegradable nanoparticles, such as inorganic nanoparticles, should be strictly restricted to therapeutic purposes due to their poorly documented safety.

Despite the difficulties, nanomedicine has achieved significant development, represented by dozens of products approved for market and hundreds in clinical trials, as well as thousands under preclinical research. A recent milestone success is Vyxeos (the liposome-encapsulated combination of daunorubicin and cytarabine) approved by FDA for acute myeloid leukemia (Alfayez et al., 2020). With the achievement of data-driven research methods including machine learning and artificial intelligence, nanoinformatics serves as a useful tool for nanomedicine design, which can manage and integrate various information including nanomaterials, manufacturing procedures, and nanotoxicity for computational prediction and design (Maojo et al., 2012; Sadan et al., 2018).

Last but not least, the availability and affordability of nanomedicines are important for society. In general, availability and affordability have been continuously improved. For example, the monthly cost of doxorubicin is $\$ 1,086$ compared to $\$ 2,311$ of its liposomal formulation (Bach and Elkin, 2020). Generic liposomes have further improved affordability.

It is expected that, with the development of nanoscience, nanotechnology-based HDACi will become a "magic bullet" against solid tumors.

\section{AUTHOR CONTRIBUTIONS}

BT wrote the draft. TL edited the manuscript. MZ and YH finalized the manuscript.

\section{FUNDING}

This work was supported by NFSC (81925035, 81673382, and 81521005), and Strategic Priority Research Program of CAS (XDA12050307), National Special Project for Significant Drugs Development (2018ZX09711002-010-002), and the Fudan-SIMM Joint Research Fund (FU-SIMM20174009).

Alfayez, M., Kantarjian, H., Kadia, T., Ravandi-Kashani, F., and Daver, N. (2020). CPX-351 (vyxeos) in AML. Leuk Lymphoma 61, 288-297. doi: 10.1080/ 10428194.2019.1660970

Alp, E., Damkaci, F., Guven, E., and Tenniswood, M. (2019). Starch nanoparticles for delivery of the histone deacetylase inhibitor CG-1521 in breast cancer treatment. Int. J. Nanomed. 14, 1335-1346. doi: 10.2147/IJN.S191837 
Amreddy, N., Babu, A., Muralidharan, R., Panneerselvam, J., Srivastava, A., Ahmed, R., et al. (2018). Chapter five - recent advances in nanoparticle-based cancer drug and gene delivery. Adv. Cancer Res. 137, 115-170. doi: 10.1016/bs. acr.2017.11.003

Bach, P., and Elkin, E. (2020). Cancer Drug Costs for A Month of Treatment at Initial Food and Drug Administration Approval. New York, NY: Center for Health Policy \& Outcomes, Memorial Sloan Kettering Cancer Center.

Bayda, S., Hadla, M., Palazzolo, S., Riello, P., Corona, G., Toffoli, G., et al. (2018). Inorganic nanoparticles for cancer therapy: a transition from lab to clinic. Curr. Med. Chem. 25, 4269-4303. doi: 10.2174/0929867325666171229141156

Bazak, R., Houri, M., El Achy, S., Kamel, S., and Refaat, T. (2015). Cancer active targeting by nanoparticles: a comprehensive review of literature. J. Cancer Res. Clin. Oncol. 141, 769-784. doi: 10.1007/s00432-014-1767-3

Bertrand, P., Blanquart, C., and Heroguez, V. (2019). The ROMP: a powerful approach to synthesize novel $\mathrm{pH}$-sensitive nanoparticles for tumor therapy. Biomolecules 9:60. doi: 10.3390/biom9020060

Chen, H., Liu, D., and Guo, Z. (2016). Endogenous stimuli-responsive nanocarriers for drug delivery. Chem. Lett. 45, 242-249. doi: 10.1248/cpb.c17-00068

Dai, Y., Xu, C., Sun, X., and Chen, X. (2017). Nanoparticle design strategies for enhanced anticancer therapy by exploiting the tumour microenvironment. Chem. Soc. Rev. 46, 3830-3852. doi: 10.1039/c6cs00592f

Danhier, F. (2016). To exploit the tumor microenvironment: since the EPR effect fails in the clinic, what is the future of nanomedicine? J. Control Release 244, 108-121. doi: 10.1016/j.jconrel.2016.11.015

Denis, I., El Bahhaj, F., Collette, F., Delatouche, R., Gueugnon, F., Pouliquen, D., et al. (2014). Vorinostat-polymer conjugate nanoparticles for Acid-responsive delivery and passive tumor targeting. Biomacromolecules 15, 4534-4543. doi: 10.1021/bm501338r

Eckschlager, T., Plch, J., Stiborova, M., and Hrabeta, J. (2017). Histone deacetylase inhibitors as anticancer drugs. Int. J. Mol. Sci. 18:1414. doi: 10.3390/ ijms 18071414

Farokhzad, O. C., and Langer, R. (2009). Impact of nanotechnology on drug delivery. ACS Nano 3, 16-20. doi: 10.1021/nn900002m

Fouladi, F., Steffen, K. J., and Mallik, S. (2017). Enzyme-responsive liposomes for the delivery of anticancer drugs. Bioconjug. Chem. 28, 857-868. doi: 10.1021/ acs.bioconjchem.6b00736

Gao, Y., Chen, Y., Ji, X., He, X., Yin, Q., Zhang, Z., et al. (2011). Controlled intracellular release of doxorubicin in multidrug-resistant cancer cells by tuning the shell-pore sizes of mesoporous silica nanoparticles. ACS Nano 5, 9788-9798. doi: 10.1021/nn2033105

Gray, M. D., Lyon, P. C., Mannaris, C., Folkes, L. K., Stratford, M., Campo, L., et al. (2019). Focused ultrasound hyperthermia for targeted drug release from thermosensitive liposomes: results from a phase I trial. Radiology 291, 232-238. doi: 10.1148/radiol.2018181445

Han, L., Wang, T., Wu, J., Yin, X., Fang, H., and Zhang, N. (2016). A facile route to form self-carried redox-responsive vorinostat nanodrug for effective solid tumor therapy. Int. J. Nanomed. 11, 6003-6022. doi: 10.2147/IJN.S118727

Hobbs, S. K., Monsky, W. L., Yuan, F., Roberts, W. G., Griffith, L., Torchilin, V. P., et al. (1998). Regulation of transport pathways in tumor vessels: role of tumor type and microenvironment. Proc. Natl. Acad. Sci. U.S.A. 95, 4607-4612. doi: 10.1073/pnas.95.8.4607

Ishii, Y., Hattori, Y., Yamada, T., Uesato, S., Maitani, Y., and Nagaoka, Y. (2009). Histone deacetylase inhibitor prodrugs in nanoparticle vector enhanced gene expression in human cancer cells. Eur. J. Med. Chem. 44, 4603-4610. doi: 10.1016/j.ejmech.2009.06.036

Jiang, W., Li, Q., Zhu, Z., Wang, Q., Dou, J., Zhao, Y., et al. (2018). Cancer chemoradiotherapy duo: nano-enabled targeting of DNA lesion formation and DNA damage response. ACS Appl. Mater. Interfaces 10, 35734-35744. doi: 10.1021/acsami.8b10901

Kim, B., Pena, C. D., and Auguste, D. T. (2019). Targeted lipid nanoemulsions encapsulating epigenetic drugs exhibit selective cytotoxicity on CDH1()/FOXM1(+) triple negative breast cancer cells. Mol. Pharm. 16, 1813-1826. doi: 10.1021/acs.molpharmaceut.8b01065

Kularatne, R. N., Washington, K. E., Bulumulla, C., Calubaquib, E. L., Biewer, M. C., Oupicky, D., et al. (2018). Histone deacetylase inhibitor (HDACi) conjugated polycaprolactone for combination cancer therapy. Biomacromolecules 19, 1082-1089. doi: 10.1021/acs.biomac.8b00221
Kumar, P., Wasim, L., Chopra, M., and Chhikara, A. (2018). Co-delivery of vorinostat and etoposide via disulfide cross-linked biodegradable polymeric nanogels: synthesis, characterization, biodegradation, and anticancer activity. AAPS PharmSciTech 19, 634-647. doi: 10.1208/s12249-017-0863-5

Kwak, T. W., Kim, D. H., Jeong, Y. I., and Kang, D. H. (2015). Antitumor activity of vorinostat-incorporated nanoparticles against human cholangiocarcinoma cells. J. Nanobiotechnol. 13:60. doi: 10.1186/s12951-015-0122-4

Lai, H., Nakase, I., Lacoste, E., Singh, N. P., and Sasaki, T. (2009). Artemisinintransferrin conjugate retards growth of breast tumors in the rat. Anticancer Res. 29, 3807-3810.

Lee, S. Y., Hong, E. H., Jeong, J. Y., Cho, J., Seo, J. H., Ko, H. J., et al. (2019). Esterase-sensitive cleavable histone deacetylase inhibitor-coupled hyaluronic acid nanoparticles for boosting anticancer activities against lung adenocarcinoma. Biomater. Sci. 7, 4624-4635. doi: 10.1039/c9bm00895k

Li, J., Gong, C., Feng, X., Zhou, X., Xu, X., Xie, L., et al. (2012). Biodegradable thermosensitive hydrogel for SAHA and DDP delivery: therapeutic effects on oral squamous cell carcinoma xenografts. PLoS One 7:e33860. doi: 10.1371/ journal.pone.0033860

Li, Y., and Seto, E. (2016). HDACs and HDAC inhibitors in cancer development and therapy. Cold Spring Harb. Perspect. Med. 6:a026831. doi: 10.1101/ cshperspect.a026831

Lin, T., Zhao, P., Jiang, Y., Tang, Y., Jin, H., Pan, Z., et al. (2016). Bloodbrain-barrier-penetrating albumin nanoparticles for biomimetic drug delivery via albumin-binding protein pathways for antiglioma therapy. ACS Nano 10, 9999-10012. doi: 10.1021/acsnano.6b04268

Liu, J., Zhao, Y., Guo, Q., Wang, Z., Wang, H., Yang, Y., et al. (2012). TATmodified nanosilver for combating multidrug-resistant cancer. Biomaterials 33, 6155-6161. doi: 10.1016/j.biomaterials.2012.05.035

Lyon, P. C., Gray, M. D., Mannaris, C., Folkes, L. K., Stratford, M., Campo, L. et al. (2018). Safety and feasibility of ultrasound-triggered targeted drug delivery of doxorubicin from thermosensitive liposomes in liver tumours (TARDOX): a single-centre, open-label, phase 1 trial. Lancet Oncol 19, 1027-1039. doi: 10.1016/S1470-2045(18)30332-2

Ma, L., Kohli, M., and Smith, A. (2013). Nanoparticles for combination drug therapy. ACS Nano 7, 9518-9525. doi: 10.1021/nn405674m

Maeda, H. (2012). Macromolecular therapeutics in cancer treatment: the EPR effect and beyond. J. Control Release 164, 138-144. doi: 10.1016/j.jconrel.2012.04.038

Maojo, V., Fritts, M., De La Iglesia, D., Cachau, R. E., Garcia-Remesal, M., Mitchell, J. A., et al. (2012). Nanoinformatics: a new area of research in nanomedicine. Int. J. Nanomed. 7, 3867-3890. doi: 10.2147/IJN.S24582

Martin, D. T., Hoimes, C. J., Kaimakliotis, H. Z., Cheng, C. J., Zhang, K., Liu, J., et al. (2013). Nanoparticles for urothelium penetration and delivery of the histone deacetylase inhibitor belinostat for treatment of bladder cancer. Nanomedicine 9, 1124-1134. doi: 10.1016/j.nano.2013.05.017

Palazzolo, S., Bayda, S., Hadla, M., Caligiuri, I., Corona, G., Toffoli, G., et al. (2018). The clinical translation of organic nanomaterials for cancer therapy: a focus on polymeric nanoparticles, micelles, liposomes and exosomes. Curr. Med. Chem. 25, 4224-4268. doi: 10.2174/0929867324666170830113755

Patra, J. K., Das, G., Fraceto, L. F., Campos, E. V. R., Rodriguez-Torres, M. D. P., Acosta-Torres, L. S., et al. (2018). Nano based drug delivery systems: recent developments and future prospects. J. Nanobiotechnol. 16:71. doi: 10.1186/ s12951-018-0392-8

Peng, H., Chen, B., Huang, W., Tang, Y., Jiang, Y., Zhang, W., et al. (2017). Reprogramming tumor-associated macrophages to reverse EGFR(T790M) resistance by dual-targeting codelivery of gefitinib/vorinostat. Nano Lett. 17, 7684-7690. doi: 10.1021/acs.nanolett.7b03756

Qiao, Y., Wan, J., Zhou, L., Ma, W., Yang, Y., Luo, W., et al. (2019). Stimuliresponsive nanotherapeutics for precision drug delivery and cancer therapy. Wiley Interdiscip. Rev. Nanomed. Nanobiotechnol. 11:e1527. doi: 10.1002/wnan. 1527

Ruttala, H. B., Ramasamy, T., Poudal, B. K., Choi, Y., Choi, J. Y., Kim, J., et al. (2017). Molecularly targeted co-delivery of a histone deacetylase inhibitor and paclitaxel by lipid-protein hybrid nanoparticles for synergistic combinational chemotherapy. Oncotarget 8, 14925-14940. doi: 10.18632/oncotarget.14742

Sadan, T., Cormode, D. P., and Popovtzer, R. (2018). Nanoinformatics revolutionizes personalized cancer therapy. Trends Cancer 4, 397-399. doi: 10.1016/j.trecan.2018.04.002 
San Jose-Eneriz, E., Gimenez-Camino, N., Agirre, X., and Prosper, F. (2019). HDAC inhibitors in acute myeloid leukemia. Cancers 11, 1794.

Seelig, A. (1998). How does P-glycoprotein recognize its substrates? Int. J. Clin. Pharmacol. Ther. 36, 50-54.

Sindhwani, S., Syed, A. M., Ngai, J., Kingston, B. R., Maiorino, L., Rothschild, J., et al. (2020). The entry of nanoparticles into solid tumours. Nat. Mater. 19, 566-575. doi: 10.1038/s41563-019-0566-2

Singleton, W. G., Collins, A. M., Bienemann, A. S., Killick-Cole, C. L., Haynes, H. R., Asby, D. J., et al. (2017). Convection enhanced delivery of panobinostat (LBH589)-loaded pluronic nano-micelles prolongs survival in the F98 rat glioma model. Int. J. Nanomed. 12, 1385-1399. doi: 10.2147/IJN.S125300

Suraweera, A., O'byrne, K. J., and Richard, D. J. (2018). Combination therapy with histone deacetylase inhibitors (HDACi) for the treatment of cancer: achieving the full therapeutic potential of HDACi. Front. Oncol. 8:92. doi: 10.3389/fonc. 2018.00092

Tak, W. Y., Lin, S. M., Wang, Y., Zheng, J., Vecchione, A., Park, S. Y., et al. (2018). Phase III heat study adding lyso-thermosensitive liposomal doxorubicin to radiofrequency ablation in patients with unresectable hepatocellular carcinoma lesions. Clin. Cancer Res. 24, 73-83. doi: 10.1158/1078-0432.CCR16-2433

Wang, E. C., Min, Y., Palm, R. C., Fiordalisi, J. J., Wagner, K. T., Hyder, N., et al. (2015). Nanoparticle formulations of histone deacetylase inhibitors for effective chemoradiotherapy in solid tumors. Biomaterials 51, 208-215. doi: 10.1016/j.biomaterials.2015.02.015

Wawruszak, A., Kalafut, J., Okon, E., Czapinski, J., Halasa, M., Przybyszewska, A., et al. (2019). Histone deacetylase inhibitors and phenotypical transformation of cancer cells. Cancers 11:E148. doi: 10.3390/cancers11020148

Weinberg, F., Ramnath, N., and Nagrath, D. (2019). Reactive oxygen species in the tumor microenvironment: an overview. Cancers 11:E1191. doi: 10.3390/ cancers 11081191

Wilhelm, S., Tavares, A. J., Dai, Q., Ohta, S., Audet, J., Dvorak, H. F., et al. (2016). Analysis of nanoparticle delivery to tumours. Nat. Rev. Mater. 1:16014.

Yang, J., Sun, X., Mao, W., Sui, M., Tang, J., and Shen, Y. (2012). Conjugate of $\mathrm{Pt}(\mathrm{IV})$-histone deacetylase inhibitor as a prodrug for cancer chemotherapy. Mol. Pharm. 9, 2793-2800. doi: 10.1021/mp200 $597 \mathrm{r}$

Yuan, Y. G., Peng, Q. L., and Gurunathan, S. (2017). Combination of palladium nanoparticles and tubastatin-A potentiates apoptosis in human breast cancer cells: a novel therapeutic approach for cancer. Int. J. Nanomed. 12, 6503-6520. doi: 10.2147/IJN.S136142

Zhang, D., Meng, Y. R., and Zhang, C. Y. (2019). Peptide-templated gold nanoparticle nanosensor for simultaneous detection of multiple posttranslational modification enzymes. Chem. Commun. 56, 213-216. doi: $10.1039 / \mathrm{c} 9 \mathrm{cc} 09019 \mathrm{c}$

Zhang, M., Liu, E., Cui, Y., and Huang, Y. (2017). Nanotechnology-based combination therapy for overcoming multidrug-resistant cancer. Cancer Biol. Med. 14, 212-227. doi: 10.20892/j.issn.2095-3941.2017.0054

Zhao, P., Wang, Y., Kang, X., Wu, A., Yin, W., Tang, Y., et al. (2018a). Dualtargeting biomimetic delivery for anti-glioma activity via remodeling the tumor microenvironment and directing macrophage-mediated immunotherapy. Chem. Sci. 9, 2674-2689. doi: 10.1039/c7sc04853j

Zhao, P., Wang, Y., Wu, A., Rao, Y., and Huang, Y. (2018b). Roles of albuminbinding proteins in cancer progression and biomimetic targeted drug delivery. Chembiochem 19, 1796-1805. doi: 10.1002/cbic.201800201

Zong, H., Shah, D., Selwa, K., Tsuchida, R. E., Rattan, R., Mohan, J., et al. (2015). Design and evaluation of tumor-specific dendrimer epigenetic therapeutics. ChemistryOpen 4, 335-341. doi: 10.1002/open.201402141

Conflict of Interest: The authors declare that the research was conducted in the absence of any commercial or financial relationships that could be construed as a potential conflict of interest.

Copyright (c) $2020 \mathrm{Tu}$, Zhang, Liu and Huang. This is an open-access article distributed under the terms of the Creative Commons Attribution License (CC BY). The use, distribution or reproduction in other forums is permitted, provided the original author(s) and the copyright owner(s) are credited and that the original publication in this journal is cited, in accordance with accepted academic practice. No use, distribution or reproduction is permitted which does not comply with these terms. 